\title{
Analysis of the effect of a root cause analysis in elderly patients with acute pancreatitis: a randomized trial
}

\author{
Xianmei Lyu ${ }^{1 \#}$, Changgui Sun ${ }^{2 \#}$, Zhixia Dong ${ }^{3}$, Yanmin $\mathrm{Wu}^{3}$ \\ ${ }^{1}$ Department of Gastroenterology, Hai'An Hospital Affiliated with Nantong Medical College, Nantong, China; ${ }^{2}$ Department of Geriatrics, Kunshan \\ Geriatric Hospital of Jiangsu Province, Suzhou, China; ${ }^{3}$ Department of Gastroenterology, the Affiliated Hospital of Jiangnan University, Wuxi, \\ China \\ Contributions: (I) Conception and design: X Lyu, Y Wu; (II) Administrative support: Z Dong, Y Wu; (III) Provision of study materials or patients: \\ X Lyu, C Sun, Y Wu; (IV) Collection and assembly of data: X Lyu, C Sun; (V) Data analysis and interpretation: X Lyu, C Sun, Z Dong; (VI) \\ Manuscript writing: All authors; (VII) Final approval of manuscript: All authors. \\ \#These authors contributed equally to this work. \\ Correspondence to: Yanmin Wu. Department of Gastroenterology, the Affiliated Hospital of Jiangnan University, Wuxi 214041, China. \\ Email: 396285432@qq.com.
}

\begin{abstract}
Background: A root cause analysis (RCA) is a structured method used to address problems. It seeks to identify the root causes of problems, and proposes solutions to achieve an ultimate goal. This study sought to investigate the effects of an RCA in treating elderly patients with acute pancreatitis (AP).

Methods: 94 patients with AP, who had been admitted to our hospital from January 2019 to January 2020, were enrolled in the study. The patients were divided into two groups using the random number table method. Each group comprised 47 patients. The control group received routine care, while the observation group underwent RCA care. The clinical efficacy, patients' negative emotions before and after the intervention, the incidence of complications, and patients' levels of satisfaction with the nursing they received were compared between the two groups.
\end{abstract}

Results: The total clinical effective rate of the observation group was higher than that of the control group $(\mathrm{P}<0.05)$. The Hamilton Anxiety Scale and Hamilton Depression Scale scores of the observation group were lower than those of the control group after the 2 -week intervention $(\mathrm{P}<0.05)$. The incidence of total complications in the observation group was lower than that in the control group $(\mathrm{P}<0.05)$. The observation group's level of satisfaction with the nursing they received was higher than that of the control group $(\mathrm{P}<0.05)$.

Conclusions: The RCA method can improve clinical efficacy, relieve negative emotion, reduce the incidence of complications, and improve the level of satisfaction with nursing of elderly patients with AP. It is worthy of promotion.

Trial registration: Chinese Clinical Trial Registry ChiCTR2100045908.

Keywords: Root cause analysis (RCA); old age; acute pancreatitis (AP); efficacy; negative emotion; complications; satisfaction

Submitted Feb 23, 2021. Accepted for publication Apr 23, 2021.

doi: 10.21037/apm-21-579

View this article at: http://dx.doi.org/10.21037/apm-21-579

\section{Introduction}

Acute pancreatitis (AP) has a high incidence of acute abdomen in clinical settings. Its main disease is the activation of pancreatic enzyme activity due to a variety of pathogenic factors (including excessive drinking and overeating, biliary diseases and alcohol abuse), which leads to severe consequences such as pancreatitis, hemorrhage, necrosis, systemic inflammatory response syndrome and multiple organ failure (1). Mild AP is usually selflimiting and has a good prognosis $(2,3)$. Moderate or 
severe pancreatitis is usually associated with persistent organ failure. About $15 \%$ of patients with mild acute pancreatitis can develop severe pancreatitis, leading to increased mortality (4). The proportion of elderly patients with model sever acute pancreatitis is higher than that of young and middle-aged people, and the mortality is also the highest. Considering that the elderly patients themselves can be combined with many basic diseases, such as diabetes, hypertension, coronary heart disease, etc., when AP occurs, the basic diseases and AP will interact, causing multiple organ damage, resulting in worse prognosis of elderly patients with AP. For such patients, nursing interventions are essential in the effective treatment of AP (4). A root cause analysis (RCA) is a structured method used to address problems. It seeks to identify the root causes of problems and proposes solutions to achieve an ultimate goal $(5,6)$. A RCA identifies the defects in a nursing system or process, and can also be used to implement corresponding measures to improve the defects in the current system or process $(5,6)$. In this study, a RCA was applied to elderly patients with AP, and the effects of the RCA on clinical efficacy, negative emotion, complication rate, and patients' level of satisfaction with nursing were examined.

We present the following article in accordance with the CONSORT reporting checklist (available at http://dx.doi. org/10.21037/apm-21-579).

\section{Methods}

\section{Patient selection}

Ninety-four patients with AP, who had been admitted to our hospital from January 2019 to January 2020, were enrolled in this study. The patients were divided into two groups using the random number table method. Each group comprised 47 patients. To be eligible to participate in this study, patients had to meet the following criteria: (I) meet the diagnostic criteria for AP, as set out in the 2014 edition of the Guidelines for Diagnosis and Treatment of Acute Pancreatitis; (II) be aged $\geq 60$; (III) have an incidence time of $\leq 72 \mathrm{~h}$; (IV) have received no other treatment before admission; (V) have signed an informed consent form or had a family member sign this form on their behalf. Conversely, patients were excluded from the study if they met any of the following exclusion criteria: (I) had other heart, liver, kidney, lung, brain or other important organ diseases; (II) had a mental disorder or a history of mental illness; (III) had serious acute and chronic infections; (IV) had malignant tumors; and/or (V) refused to cooperate with this research. The study was approved by the Ethics Committee of the Affiliated Hospital of Jiangnan University (KY2018168), and conducted in accordance with the Declaration of Helsinki (as revised in 2013). All patients provided informed consent.

\section{Interventions}

The control group underwent a routine nursing intervention whereby intervention staff educated patients on AP-related knowledge, informed patients and their families that they needed to actively cooperate with doctors' treatment and the nursing work of the nursing staff, and provided patients with psychological counseling to eliminate their negative psychological emotions. In addition to being provided with the same routine nursing as that provided to the control group, the observation group also underwent a RCA intervention whereby: (I) they were treated by an established intervention group; (II) the root cause was identified; (III) improvement measures were formulated.

The team members of the established intervention group comprised senior and experienced medical staff, including specialists, a head nurse, and specialist nurses. A head nurse was the team leader, and all members of the team underwent knowledge training on RCA. All the intervention personnel had to pass an assessment before implementing the RCA intervention. The intervention group summarized and analyzed previous adverse nursing events in AP, and identified the root causes of adverse events using a brainstorming method. The following three points were identified: (I) the patients had insufficient knowledge of catheterization; (II) the medical staff did not implement adequate preventive measures against complications; and (III) the number of nurses on duty at night was low.

In relation to the formulation of improvement measures, a system was first developed to prevent catheters from falling off. The intervention team selected high-risk patients based on the "Catheter Evaluation and Monitoring Mechanism" and the actual clinical conditions of the hospital. Scores were calculated based on the following two aspects: (I) level of awareness: 1 point was awarded to those with clear awareness, 2 points to those who were unwilling to cooperate with treatment or had a history of sedative drug use before, 3 points to those with vague consciousness, and 4 points to those with irritability; (II) catheter type: 1 point was awarded to those who had indwelling Class III catheters, such as an infusion tube, stomach tube or oxygen 
inhalation tube, 2 points to those who had indwelling Class II catheters, such as peripheral vein indwelling needle, negative pressure balloon, and deep vein catheter, 3 points to those who had indwelling Class I catheters, such as tracheal cannula, arteriovenous cannula, thoracic tube and ventricular drainage tube. Patients with a score of $\leq 2$ were classified as low-risk patients, patients with a score of 3 to 5 were classified as intermediate-risk patients, and patients with a score of $\geq 5$ were classified as high-risk patients. Lowrisk patients received a catheter assessment once a week, intermediate-risk patients once every 3 days, and high-risk patients once a day.

Second, a publicity and education system was developed for patient infusion risk management. Interveners explained the infusion process to patients and their families in detail, informed patients of the correct method of fist clenching, encouraged patients to express their inner worries and concerns, and provided answers systematically. After successful puncture, the intervention staff thanked the patients for their cooperation, encouraged them to actively receive treatment, quickly established an effective venous channel to maintain the effective circulating blood volume and water-electrolyte acid-base balance, and closely monitored the dehydration status and cardiopulmonary function of the patients, continuously adjusting the infusion speed.

Third, a scientific scheduling system was developed. When the head nurse scheduled the nurses on duty, midshift nurses' working hours were appropriately extended, night shift nurses were scheduled to commence work half an hour earlier to increase the number of night shift nurses, and the number of nurses on duty were increased to monitor changes in patients' body temperature, blood pressure, blood gas and respiration.

\section{Evaluation indexes}

\section{Clinical efficacy}

Clinical efficacy was graded as either markedly effective, effective, or ineffective. It was graded as markedly effective, if after 2 weeks of intervention, the clinical symptoms of patients were significantly reduced, and all indicators had essentially returned to normal. It was graded as effective, if after 2 weeks of intervention, the clinical symptoms of patients were relieved and the indexes were recovered. It was graded as ineffective, if after 2 weeks of intervention, the patients' clinical symptoms and indicators did not improve or even worsened. The total effective rate was calculated using the following formula: (effective number + effective number)/total number $\times 100 \%(7)$.

\section{Negative emotions}

The Hamilton Anxiety Scale (HAMA) and Hamilton Depression Scale (HAMD) were used to evaluate the anxiety and depression of patients in the two groups before and after the 2-week intervention. HAMA scale has good reliability and validity, the total score reliability coefficient $\mathrm{r}$ is 0.93 , the reliability coefficient of each single symptom score is $0.83-1.00$, the validity coefficient is 0.36 . There are 14 items, each item $0-4$ points, $>7$ points indicates that there may be anxiety, the higher the score, the more serious the anxiety (8). HAMD scale has good reliability and validity, the total score evaluation reliability coefficient $r$ is 0.99 , the reliability coefficient of each single symptom score is $0.78-0.98$, the validity coefficient is 0.37 , there are 17 items, each item $0-4$ points, $>7$ points indicates that there may be depression, the higher the score, the more serious the depression (8).

\section{The incidence of complications}

The number of cases of shock, heart damage, infection, renal failure, acute respiratory distress syndrome, and digestive system complications in the two groups in the 2 -week intervention period were counted, and the total incidence of complications was calculated.

\section{Nursing satisfaction}

A nursing satisfaction questionnaire was developed and used to evaluate the satisfaction of patients in the two groups after the 2-week intervention. The questionnaire comprised 10 items, each of which was scored from $0-10$ points. A total score could range from $0-100$ points. A score of $85-$ 100 points indicated that a patient was very satisfied, a score of 70-84 points that the patient was generally satisfied, and a score of $<70$ points that the patient was not satisfied. Satisfaction was calculated using the following formula: (very satisfied number + generally satisfied number)/total number $\times 100 \%$.

\section{Statistical analyses}

The statistical analysis in this study was performed using SPSS 20.0 (SPSS Inc., Chicago, IL, USA). The HAMA and HAMD scores of the two groups before and after the intervention were expressed as mean \pm standard deviation $(\bar{x} \pm s)$, and compared using 2 -tailed $t$-tests. The two groups' 


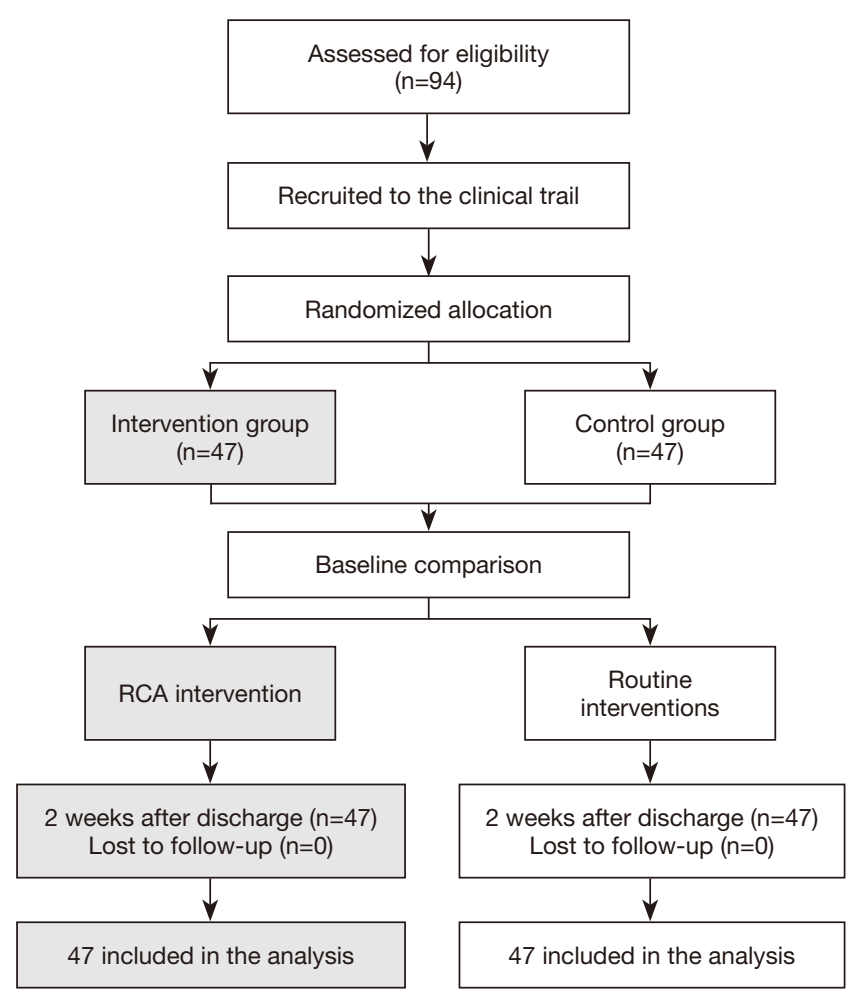

Figure 1 Study profile.

clinical efficacy, incidence of complications, and levels of satisfaction with nursing were expressed by rates and percentages, and compared using the chi-squared $\left(\chi^{2}\right)$ test or rank-sum test. A P value of $<0.05$ was considered statistically significant.

\section{Results}

\section{General information}

This study comprised 94 elderly patients with AP. The control group included 27 males and 20 females, aged $61-80$ years old, with an average age of $69.27 \pm 3.28$. The time from onset to admission was 5-52 hours with an average time of $22.82 \pm 4.84$ hours. In relation to level of education, 7 patients had a high school or above level of education, 18 patients had a junior high school level of education, and 22 patients had an elementary school or below level of education. In relation to marital status, 27 patients were married, and 20 were unmarried/divorced/ widowed. In relation to family income, 5 patients earned less than 4,000 yuan per month, 22 patients earned 4,0008,000 yuan per month, and 20 patients earned more than
8,000 yuan per month.

The observation group comprised 28 males and 19 females, aged 61-79 years old, with an average age of $69.55 \pm 3.15$. The time from onset to admission was $6-54$ hours with an average time of $21.96 \pm 4.73$ hours. In relation to level of education, 6 patients had a high school or above level of education, 17 patients had a junior high school level of education, and 24 patients had an elementary school or below level of education. In relation to marital status, 25 patients were married, and 22 were unmarried/ divorced/widowed. In relation to family income, 5 patients earned less than 4,000 yuan per month, 23 patients earned 4,000-8,000 yuan per month, and 19 patients earned more than 8,000 yuan per month.

There were no statistically significant differences between the two groups of patients in terms of general data, such as gender, age, time from onset to admission, education level, marital status, or family income $(\mathrm{P}>0.05)$. (see Figure 1, Table 1).

\section{Clinical efficacy}

In relation to the observation group, 30 cases were markedly effective, 16 cases were effective, and 1 case was ineffective (total effective rate: $97.87 \%$ ). In relation to the control group, 22 cases were markedly effective, 15 cases were effective and 10 cases were ineffective (total effective rate: $78.72 \%$ ). Comparing the two groups, the total clinical effective rate in the observation group was higher than that in the control group, and the difference was statistically significant $\left(\chi^{2}=8.340, P=0.004\right)$. See Table 2 for details.

\section{Negative emotions}

Before the intervention, there were no statistical differences in the HAMA and HAMD scores of the two groups $(t=0.063$, 0.104, $\mathrm{P}=0.950,0.918$ ). After the 2 -week intervention, the HAMA and HAMD scores in the observation group were lower than those in the control group, and the difference was statistically significant $(\mathrm{t}=8.658,8.265, \mathrm{P}=0.000,0.000)$. See Table 3 for details.

\section{The incidence of complications}

In the observation group, there were 1 case of shock, 1 case of heart damage, 2 cases of renal failure, 2 cases of acute respiratory distress syndrome, and 4 cases of digestive system complications (total complication rate: $21.28 \%$ ). In 
Table 1 Comparison of general information between the two groups (n, \%)

\begin{tabular}{|c|c|c|c|c|}
\hline Variables & Control group $(n=47)$ & Observation group $(n=47)$ & Statistic value & $P$ value \\
\hline Time from onset to admission (hours) & $22.82 \pm 4.84$ & $21.96 \pm 4.73$ & $t=0.871$ & 0.386 \\
\hline Sex & & & $\chi^{2}=0.044$ & 0.834 \\
\hline Male & $27(57.45)$ & $28(59.57)$ & & \\
\hline Education level & & & $\chi^{2}=0.193$ & 0.908 \\
\hline High school and above & $7(14.89)$ & $6(12.77)$ & & \\
\hline Junior high school & $18(38.30)$ & $17(36.17)$ & & \\
\hline Elementary school and below & $22(46.81)$ & $24(51.06)$ & & \\
\hline Unmarried/divorced/widowed & $20(42.55)$ & $22(46.81)$ & & \\
\hline Family income (yuan) & & & $\chi^{2}=0.048$ & 0.976 \\
\hline$<4,000$ & $5(10.64)$ & $5(10.64)$ & & \\
\hline $4,000-8,000$ & $22(46.81)$ & $23(48.94)$ & & \\
\hline$>8,000$ & $20(42.55)$ & $19(40.43)$ & & \\
\hline
\end{tabular}

Table 2 Comparison of clinical efficacy between the two groups (n, \%)

\begin{tabular}{|c|c|c|c|c|}
\hline Variables & Control group $(n=47)$ & Observation group $(n=47)$ & $\chi^{2}$ value & $P$ value \\
\hline Effective & $15(31.91)$ & $16(34.04)$ & - & - \\
\hline Ineffective & $10(21.28)$ & $1(2.13)$ & - & - \\
\hline Total effective rate & 37 (78.72) & $46(97.87)$ & 8.34 & 0.004 \\
\hline
\end{tabular}

Table 3 Comparison of negative emotions between the two groups before and after intervention $(\bar{x} \pm s$, points)

\begin{tabular}{lccc}
\hline Time & Group & HAMA score & HAMD score \\
\hline Before the intervention & Control group $(n=47)$ & $14.17 \pm 3.05$ & $15.71 \pm 3.25$ \\
& Observation group $(n=47)$ & $14.21 \pm 3.11$ & $15.64 \pm 3.28$ \\
& $t$ value & 0.063 & 0.104 \\
& P value & 0.950 & 0.918 \\
After the intervention & Control group $(n=47)$ & $11.42 \pm 2.89$ & $12.03 \pm 3.12$ \\
& Observation group $(n=47)$ & $6.81 \pm 2.23$ & $7.35 \pm 2.31$ \\
& $t$ value & 8.658 & 8.265 \\
\hline
\end{tabular}


Table 4 Comparison of the incidence of complications between the two groups (n, \%)

\begin{tabular}{|c|c|c|c|c|}
\hline Complications & Control group $(n=47)$ & Observation group $(n=47)$ & $\chi^{2}$ value & $P$ value \\
\hline Heart damage & $2(4.26)$ & $1(2.13)$ & 0.344 & 0.557 \\
\hline Infection & $2(4.26)$ & $0(0.00)$ & 0.511 & 0.475 \\
\hline Renal failure & $5(10.64)$ & $2(4.26)$ & 0.617 & 0.432 \\
\hline Digestive system complications & $7(14.89)$ & $4(8.51)$ & 0.927 & 0.336 \\
\hline Total complications & $25(53.19)$ & $10(21.28)$ & 10.242 & 0.001 \\
\hline
\end{tabular}

Table 5 Comparison of patients' level of satisfaction with nursing between the two groups (n, \%)

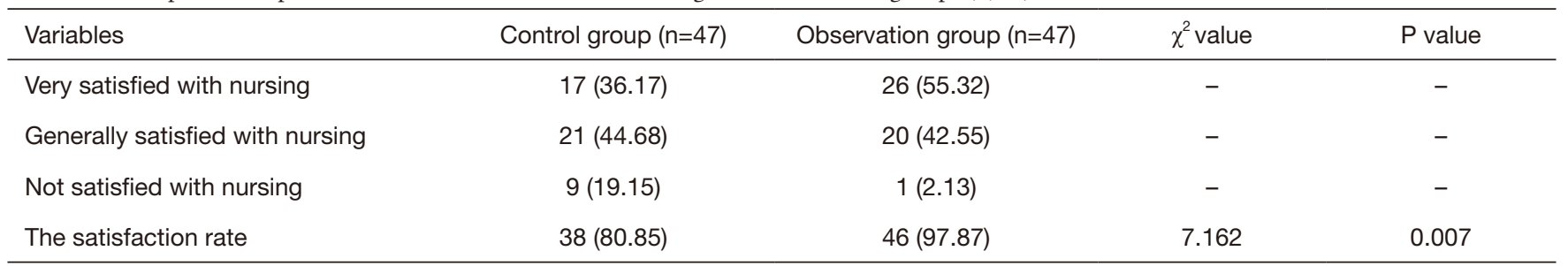

the control group, there were 3 cases of shock, 2 cases of heart damage, 2 cases of infection, 5 cases of renal failure, 6 cases of acute respiratory distress syndrome, and 7 cases of digestive system complications (total complication rate: $53.19 \%$ ). Compared to the control group, the total incidence of complications in the observation group was lower, and the difference was statistically significant $\left(\chi^{2}=10.242, P=0.001\right)$. See Table 4 for details.

\section{Level of satisfaction with nursing}

In the observation group, 26 patients were very satisfied with the nursing, 20 patients were generally satisfied, and 1 patient was not satisfied (total satisfaction rate: $97.87 \%$ ). In the control group, 17 patients were very satisfied with the nursing, 21 patients were generally satisfied, and 9 patients were not satisfied (total satisfaction rate: $80.85 \%$ ). The observation groups' level of satisfaction with the nursing was higher than that of the control group, and the difference was statistically significant $(\mathrm{P}<0.05)$. See Table 5 for details.

\section{Discussion}

$\mathrm{AP}$ is a one of the common acute abdominal diseases in clinical settings. It is characterized by a dangerous condition and rapid progress. In severe cases, many complications, such as organ failure, shock and pseudocyst, may occur (9). Severe AP (SAP), which is also known as acute hemorrhagic necrotizing pancreatitis, is characterized by many complications and high mortality. Patients with SAP experience an inflammatory reaction, hemodynamic changes, and metabolic disorders in the early stage, and with the development of the disease, severe complications, such as severe infection, electrolyte disturbance, deep venous thrombosis of lower limbs, multiple organ failure, and pancreatic encephalopathy, may occur $(10,11)$. Studies had shown that the mortality rate of patients with SAP is as high as $70 \%$. Thus SAP poses a serious threat to patients' lives. Consequently, it is very important that effective and scientific nursing interventions are implemented early to reduce the occurrence of complications of AP, and slow down the development of the disease (12). With the improvement of people's quality of life, the nursing requirements of patients and their families have gradually improved. In addition, the physical function of elderly patients with AP is declining, which requires close attention from medical staff (13). In this study, a RCA was used to conduct an intervention with elderly patients with $\mathrm{AP}$, and remarkable 
results were achieved.

The results of this study showed that the total clinical effective rate of the observation group was higher than that of the control group $(\mathrm{P}<0.05)$. Further, the RCA intervention effectively improved the clinical efficacy of elderly patients with AP. The RCA intervention ensured that patients received improved treatment. Further, the medical abilities of the staff were strengthened, as an intervention team was established to summarize and analyze the problems in the treatment of elderly patients with $\mathrm{AP}$, and formulate detailed targeted measures $(14,15)$.

The results of this study also showed that patients' HAMA and HAMD scores in the observation group were lower than those in the control group after the 2-week intervention $(\mathrm{P}<0.05)$. Thus, RCA effectively improved patients' negative emotions. The main reasons for these results were that the interveners explained the purpose and significance of the intervention in detail before the RCA intervention, affirmed the patients' attitudes of active cooperation, identified the causes of patients' negative emotions, and provided timely targeted counseling to help them eliminate these negative emotions $(16,17)$. Further, the patients actively cooperated with the medical staff in the treatment process, which may have sped up patients' recovery from their illness, enhanced patients' confidence in treating diseases and established a positive psychology.

The total complication rate of the observation group was lower than that of the control group, and their level of satisfaction with the nursing was higher than that of the control group $(\mathrm{P}<0.05)$. There appear to be 4 main reasons for these results. First, the implementation of a reasonable scheduling system enabled the interveners to observed dynamic changes in patients' conditions in real time, and the interveners provided different preventive interventions at different times. Second, the RCA effectively prevented adverse nursing events from occurring, and enhanced nursing staff's awareness of prevention $(18,19)$. Third, the intervention model of RCA emphasized cooperation between medical staff, which enabled them to find and address changes in patients' illness in time. Finally, the RCA intervention model not only enhanced cooperation between medical staff, but also solved problems related to insufficient inspections and the low number of night shift nursing staff. Notably, following the RCA intervention, nursing staff training was strengthened, and a scientific scheduling system was formulated, which effectively reduced the occurrence of complications and improved patients' and their families' levels of satisfaction with nursing (20).

\section{Conclusions}

The RCA method appears to improve clinical efficacy, relieve patients' negative emotions, reduce the incidence of complications, and improve elderly AP patients' levels of satisfaction with nursing. It is worthy of promotion.

\section{Acknowledgments}

Funding: None.

\section{Footnote}

Trial Protocol: Available at http://dx.doi.org/10.21037/apm$21-579$

Reporting Checklist: The authors have completed the CONSORT reporting checklist. Available at http://dx.doi. org/10.21037/apm-21-579

Data Sharing Statement: Available at http://dx.doi. org/10.21037/apm-21-579

Conflicts of Interest: All authors have completed the ICMJE uniform disclosure form (available at http://dx.doi. org/10.21037/apm-21-579). The authors have no conflicts of interest to declare.

Ethical Statement: The authors are accountable for all aspects of the work, including ensuring that questions related to the accuracy or integrity of any part of the work were appropriately investigated and resolved. The study was approved by the Ethics Committee of the Affiliated Hospital of Jiangnan University (KY2018-168), and conducted in accordance with the Declaration of Helsinki (as revised in 2013). All patients provided informed consent.

Open Access Statement: This is an Open Access article distributed in accordance with the Creative Commons Attribution-NonCommercial-NoDerivs 4.0 International License (CC BY-NC-ND 4.0), which permits the noncommercial replication and distribution of the article with the strict proviso that no changes or edits are made and the original work is properly cited (including links to both the formal publication through the relevant DOI and the license). See: https://creativecommons.org/licenses/by-nc-nd/4.0/. 


\section{References}

1. Li J, Meng L, Zhang H, et al. Efficacy and drug resistance of antibacterials in patients' with acute pancreatitis and stress ulcer bleeding. Pak J Pharm Sci 2017;30:1905-9.

2. Singh S, Eguchi M, Min SJ, et al. Outcomes of patients with cancer discharged to a skilled nursing facility after acute care hospitalization. J Natl Compr Canc Netw 2020;18:856-65.

3. Dong Z, Song J, Ge M, et al. Effectiveness of a multidisciplinary comprehensive intervention model based on the Hospital Elderly Life Program to prevent delirium in patients with severe acute pancreatitis. Ann Palliat Med 2020;9:2221-8.

4. Herman JM, Kitchen H, Degboe A, et al. Exploring the patient experience of locally advanced or metastatic pancreatic cancer to inform patient-reported outcomes assessment. Qual Life Res 2019;28:2929-39.

5. Lin HF, Liao KF, Chang CM, et al. Association of use of selective serotonin reuptake inhibitors with risk of acute pancreatitis: a case-control study in Taiwan. Eur J Clin Pharmacol 2017;73:1615-21.

6. Boije K, Drocic A, Engström M, et al. Patients' Perceptions of Experiences of Recovering From Acute Pancreatitis An Interview Study. Gastroenterol Nurs 2019;42:233-41.

7. Karpavicius A, Dambrauskas Z, Gradauskas A, et al. The clinical value of adipokines in predicting the severity and outcome of acute pancreatitis. BMC Gastroenterol 2016;16:99.

8. Michael N, Beale G, O'Callaghan C, et al. Timing of palliative care referral and aggressive cancer care toward the end-of-life in pancreatic cancer: a retrospective, singlecenter observational study. BMC Palliat Care 2019;18:13.

9. Siepsiak M, Szałek E, Karbownik A, et al. Pharmacokinetics of paracetamol in patients with chronic pancreatitis. Pharmacol Rep 2016;68:733-6.

10. Ozdemir BA, Sinha S, Karthikesalingam A, et al. Mortality of emergency general surgical patients and associations with hospital structures and processes. Br J Anaesth 2016;116:54-62.

11. Ho TW, Wu JM, Kuo TC, et al. Change of Both

Cite this article as: Lyu X, Sun C, Dong Z, Wu Y. Analysis of the effect of a root cause analysis in elderly patients with acute pancreatitis: a randomized trial. Ann Palliat Med 2021;10(5):57385745. doi: 10.21037/apm-21-579
Endocrine and Exocrine Insufficiencies After Acute Pancreatitis in Non-Diabetic Patients: A Nationwide Population-Based Study. Medicine (Baltimore) 2015;94:e1123.

12. Thu Huynh V, Bergeron S. Asparaginase toxicities: identification and management in patients with acute lymphoblastic leukemia. Clin J Oncol Nurs 2017;21:E248-59.

13. Wen Y, Han C, Liu T, et al. Chaiqin chengqi decoction alleviates severity of acute pancreatitis via inhibition of TLR4 and NLRP3 inflammasome: Identification of bioactive ingredients via pharmacological sub-network analysis and experimental validation. Phytomedicine 2020;79:153328.

14. Rosołowski M, Lipiński M, Dobosz M, et al. Management of acute pancreatitis (AP)-Polish Pancreatic Club recommendations. Prz Gastroenterol 2016;11:65-72.

15. Tian F, Li H, Wang L, et al. The diagnostic value of serum C-reactive protein, procalcitonin, interleukin-6 and lactate dehydrogenase in patients with severe acute pancreatitis. Clin Chim Acta 2020;510:665-70.

16. Shen QX, Xu GX, Shen MH. Effect of early enteral nutrition $(\mathrm{EN})$ on endotoxin in serum and intestinal permeability in patients with severe acute pancreatitis. Eur Rev Med Pharmacol Sci 2017; 21:2764-8.

17. Perito ER, Lowe ME, Schwarzenberg SJ. Predicting Severity in Acute Pancreatitis in Children: What Can a Quantitative Score Add to Clinical Judgement. J Pediatr Gastroenterol Nutr 2020;71:419-20.

18. Jiang X, Zheng YW, Bao SH, et al. Drug discovery and formulation development for acute pancreatitis. Drug Deliv 2020;27:1562-80.

19. Komara NL, Paragomi P, Greer PJ, et al. Severe acute pancreatitis: capillary permeability model linking systemic inflammation to multiorgan failure. Am J Physiol Gastrointest Liver Physiol 2020;319:G573-83.

20. Konur S, Ozkahraman A, Surmeli N, et al. The Severity of Acute Pancreatitis According to Modified Balthazar Classification in Patients With Pancreatic Cancer. Tumori 2020;106:356-61.

(English Language Editor: L. Huleatt) 